\title{
Signal Processing Mechanism for Power Grid Monitoring based on Event Rule
}

\author{
Qiang Song ${ }^{1}$, Hong Gong ${ }^{1}$, Jiaqiao Zhao ${ }^{1}$, Liqiu Yan ${ }^{1}$, Shugang Yao ${ }^{1}$, \\ Jingfeng Zhang ${ }^{1}$ \\ ${ }^{1}$ State Grid Jining Power Supply Company, Power Transmission \& Transformation Engineering \\ Construction Department, Jining Shandong, 272000
}

Keywords: Rule; Power Grid Monitoring; Signal Classification; Event Processing

\begin{abstract}
Optimizing and screening signals sent from useful power grid equipment is beneficial for monitoring personnel to judge the fault situations of field equipment more quickly and correctly, therefore, optimizing centralized monitoring signals of power grid is a very important work. In this Paper, monitoring signals of substation operation are classified, problems in protection signal monitoring are summarized and improvement measures for signal monitoring are proposed, which is helpful for the implementation of centralized monitoring of power grid, more convenient for operating personnel to judge the accuracy of key abnormal information in system and find out the fault cause more quickly so as to propose processing suggestions to promote the efficiency of staff in treating accident; besides, it provides a more convenient support for the intelligent query of alarm message and is helpful for checking and analyzing the operation of system so that the scalability and response speed of intelligent alarm system are greatly improved.
\end{abstract}

\section{Introduction}

In order to maintain the stability and sustainable development of society, not only shall energy crisis be dealt with, but also the power system shall be ensured in pretty stable operation; more intelligent design of regional power grid will become one of the important targets of design and development of power grid, and at the same time, to ensure the safe operation of power grid, realizing the comprehensive alarm system will become of great importance; to monitor and ensure the safe operation of power grid, comprehensive alarm system of monitoring is a very effective tool. To comprehensively and intelligently analyze the relations between remote signaling and telemetry information, information including faults can be filtered mutely and effectively so that scope of fault can be speculated quickly. To observe and analyze the correlation of alarm system will be helpful for operating personnel to determine the fault source correctly, reduce processing response time and improve the reliability of network. According to the present situations, many literatures are involved in the matters to make some problems in comprehensive alarm system of power grid monitoring solved, but there is still a lack of perfect innovative design thoughts and implementation methods.

\section{Category of Monitoring Signals of Substation Operation}

Based on the urgent degree of signal processing and signal characteristics, the switch signals monitored are classified into five categories, which is displayed in monitoring system.

(1) Accident signals (category A)

Accident signals include accident reflecting signals and circuit breaker deflection signal: 1) switch on \& switch off of circuit breaker; 2) protection action signal; 3) cell accident signal; 4) energy dis-storage signal of circuit breaker spring and switch on \& off of circuit breaker are important monitoring signals and they are listed into category A for enhancing focus; energy dis-storage signal of circuit breaker spring will be accompanied with closed circuit breaker, which can be used as the basis for reclosing motion at accident trip.

(2) Alarm signals (category B) 
As for power-loss, lockout, abnormal, alarm and other signals related to equipment, they shall be focused on immediately and processed. 1) alarm signals of circuit breaker mechanism: switch on \& off lockout, SF6 alarm lockout, nitrogen leakage alarm, local control signal, loss of heat and energy-storage power source, etc.;2) abnormal signals of protection equipment; 3) secondary circuit alarm signal; 4) unlocking signal; 5) other abnormal alarm signals; 6) analogue threshold-crossing signal.

(3) Deflection signals (category C)

Deflection signals are mainly deflection signals of isolating switch and grounding-knife switch, which shall be focused on in switching operation.

(4) Prompt signals (category D)

Ordinary prompt signals are not required to be processed. In VQC automatic regulation, capacitor, switch on \& off of reactor breaker, main transform junction gear changes, etc. shall all be listed into signals of category D, so as to reduce monitoring workload.

(5) Automation equipment status signals (category E)

The signals can reflect the working conditions of automation equipment through which signal message can be sent and uploaded.

\section{Exact Orientation of Various Signals}

Seen from above table, frequent signals of system are various alarm signals; when line trips or is grounded, plenty of alarm and return signals are accompanied and are intertwining with normal monitoring information, which goes against important information monitoring. How to classify information, filter missent and dither signals is the key of exact orientation of information; through discussions with professional staff in telecontrol, protection and communication, we classify information into following six types:

The first type: accident trip signals of switch protection action;

The second type: $35 \mathrm{kV}$ and $10 \mathrm{kV}$ bus grounded signals;

The third type: switch pressure reduction block (GIS equipment: alarm for low pressure in air chamber), control circuit break, SF6 rheological pressure reduction signal, voltage transformer and current transformer break;

The fourth type: signal of abnormal protection equipment and equipment fault;

The fifth type: switch mechanism: (energy dis-storage spring; hydraulic mechanism pressure reduction lockout);

The sixth type: no data of channel

As for other types of signals, only event and voice are required to be sent while alarm is needless to be sent; for events, abnormal conditions and endangering signals affecting safe operation of equipment are connected to alarm bell for maintaining \& conducting manual reset, which are the measures for solving monitoring misses; "big operation” control has many substations and few personnel, easily leading to monitoring misses during exhaustion or nightwork of personnel, therefore, monitoring equipment shall be connected to alarm bell and its manual reset function shall be maintained and conducted.

As a result of computer networks, channels, equipment and other factors, missent protection information, useless information and amount of information in the SOE account for a large proportion, to further optimize the signal and eliminate the false and retain the true are required, thus realizing that all messages sent by monitoring are all useful information. First of all, we contact professional personnel of protection and automation, etc. to discuss which signals can be deleted and which signals can be not in voice prompt, so as to optimize the alarm information classification, meanwhile, professional personnel of automation and network information try to strengthen the maintenance on network, thus avoiding the occurrences of missending and sending omissions; second, for some frequent signals, appropriate delay actions and sending in several seconds delay after action can be taken, for example: for instantaneous grounded fault signal of power grid, due to the particularity of instantaneous grounded fault of power grid, monitoring personnel shall be reminded after the first sending of signal, and then the grounded fault message within 3 seconds can 
be shielded, thus reducing amount of signals; third, in protection signals, dispensable information is not displayed any more, for example, in GPS time setting, fault detection's action reset, and such types of signals are only with message displays and without voice prompts; at last, repair and maintenance personnel are urged to improve disappearing rate to ensure monitoring system be in the best state, the less defect information there are, the better the state will become.

\section{Comprehensive Alarm System of Regional Power Grid Monitoring}

(1) Principle for design of normal operation alarm system

At present, although automation alarm system of power grid dispatching can be displayed in alarm column, the causes of emergency situations can not be clearly distinguished yet, and whether it is caused as a result of accident switch deflection or normal switch deflection can not be judged; therefore, it is required to distinguish the two in accordance with their different properties in design. If it is an accident switch deflection and maintenance signal is transmitted for a long distance at the same time, a special alarm signal will be sent in alarm column; if it is a normal operation alarm, in general, the alarm is in standard alarm sound, and indicator light blinks will occur in alarm column, which will stop blinking immediately once it is confirmed.

(2) Principle for alarm design of voltage exceeding limits

The principle for alarm design of voltage exceeding limits can be judged according to the font color, in which: green is a symbol of normal value, pink is a symbol of exceeding upper limit, red is a symbol of exceeding the very upper limit, blue is a symbol of exceeding lower limit and yellow is a symbol of exceeding the very lower limit. When limit is exceeded in regional power grid, in order to remind operating personnel of the occurring phenomenon, the color kalor of voltage bar will be changed. When system is in very low voltage, insufficient power and is mainly operated through recombination of motor, motor will act slowly due to insufficient load; many reactive powers are extracted from the system, which will finally lead to the lack of reactive power, and voltage will be maintained at a very low level and can not reach the original level for long. Supposing there is no effective method to control such phenomena, it will be a vicious cycle of keeping voltage of power grid at reduced level, which will finally lead to voltage collapse. At this point, power supply including reactive power supply shall be provided with advanced consciousness in its construction process, reactive power management shall also be further improved. At present, unattended substation is gradually increasing, and at the same time, comprehensive monitoring equipment of voltage is also being designed and promoted, where some accidents will occur, such as right age of equipment, etc., therefore, comprehensive monitoring equipment of power grid voltage shall be paid attention to and to make voltage collapse vanished before it comes into being shows the urgency and importance of intelligent alarm design of voltage.

(3) Alarm information processing

In alarm information list, classification, grading, storage processing, decision and real-time status are required to be analyzed and recorded, etc., therefore, any alarm information shall be with a processing process; by processing these information cautiously, above functions can be realized, which can be combined into a processing module of alarm information. Fault information show different influence degrees on system, therefore, they are classified into different types according to the types of occurring faults; alarm information can be classified into three different levels: (1) prompt alarm: only plays a role of prompt; it sends alarm information to the operator on duty at that moment and prompts operation information at the moment; (2) general alarm: will not affect the normal operation of system, and only locally or singly influence the system; (3) emergency alarm: makes system can not be in stable operation any more and has direct influence on the safety of system. Another graphic alarm method is to show different levels of alarm information by using hierarchical displays and different colors, and arrange these alarm information in order, for example, accident alarm information are arranged from top to low according to the levels, or from near to far according to the time sequence of accident occurrence. 


\section{Conclusion}

In this Paper, comprehensive voltage reactive power control design for comprehensive alarm intelligent system of the regional power grid monitoring is proposed, which proposes design methods for alarm at normal operation and alarm of voltage exceeding limits, and helps a deeper understanding of processing alarm information at the same time. In this way, not only dispatch operating personnel can be prompted for prevention of accident, but also the potential hazard of power grid collapse can be removed, reducing the processing time of accident, making it more convenient for operating personnel to judge the accuracy of key abnormal information in system and find out the fault cause, so that processing suggestions can be proposed and efficiency of staff in processing accident is improved; besides, it provides convenient supports for the intelligent query of alarm information, is helpful for checking and analyzing the operation situation of system and makes the scalability and response speed of intelligent alarm system greatly improved.

\section{References}

[1] Giannakis G B, Kekatos V, Gatsis N, et al. Monitoring and Optimization for Power Grids: A Signal Processing Perspective[J]. IEEE Signal Processing Magazine, 2013, 30(5):107-128.

[2] Silva L R M, Duque C A, Ribeiro P F. Smart signal processing for an evolving electric grid[J]. EURASIP Journal on Advances in Signal Processing, 2015, 2015(1):1-13.

[3] Gu I Y H, Bollen M H J, Le C D. Signal Processing And Classification Tools For Intelligent Distributed Monitoring And Analysis Of The Smart Grid[C]// IEEE Pes International Conference and Exhibition on Innovative Smart Grid Technologies. IEEE, 2011:1-7.

[4] Shi W, Zhu Y, Huang T, et al. An Integrated Data Preprocessing Framework Based on Apache Spark for Fault Diagnosis of Power Grid Equipment[J]. Journal of Signal Processing Systems, 2016:1-16.

[5] Qiu M, Gao W, Chen M, et al. Energy Efficient Security Algorithm for Power Grid Wide Area Monitoring System[J]. Smart Grid IEEE Transactions on, 2011, 2(4):715-723. 